Balzac, l'éternelle genèse, textes réunis et présentés par Jacques Neefs, Saint-Denis, Presses universitaires de Vincennes, coll. « Manuscrits modernes », 2015, $256 \mathrm{p}$.

\title{
Lionel Rérat
}

\section{(2) OpenEdition}

\section{Journals}

Édition électronique

URL : http://journals.openedition.org/genesis/1569

DOI : 10.4000/genesis.1569

ISSN : 2268-1590

Éditeur :

Presses universitaires de Paris Sorbonne (PUPS), Société internationale de génétique artistique littéraire et scientifique (SIGALES)

\section{Édition imprimée}

Date de publication : 27 novembre 2015

Pagination : 204-206

ISBN : 9791023105049

ISSN : 1167-5101

\section{Référence électronique}

Lionel Rérat, "Balzac, l'éternelle genèse, textes réunis et présentés par Jacques Neefs, Saint-Denis, Presses universitaires de Vincennes, coll. «Manuscrits modernes », 2015, 256 p. », Genesis [En ligne], 41 | 2015, mis en ligne le 05 mai 2017, consulté le 22 septembre 2020. URL : http:// journals.openedition.org/genesis/1569; DOI : https://doi.org/10.4000/genesis.1569 
avec le support d'écriture. Sans doute peut-on regretter leur intégration sans précaution dans le Journal, comme cela a été fait dans la dernière édition Pléiade, qui induit une perspective de lecture trompeuse. Et on concèdera que la difficulté éditoriale est de taille. Il faut en effet combiner deux ensembles de contraintes qui apparaissent exclusives : ces notes se caractérisent par une certaine unité formelle et sont situées, par la datation, en relation au cours de l'existence du scripteur ; mais d'un autre côté, leur dispersion sur les différents volumes ainsi que le rapprochement de certaines d'entre elles sur un même support n'est pas non plus sans importance. Une édition uniquement numérique peut paraître une solution à la mesure de la difficulté, mais cela n'est pas entièrement cohérent avec l'entreprise des Journaux et papiers qui complète très utilement le site <manuscrits-de-stendhal. org $>-$ dont il faut souligner l'intérêt et la qualité. La maniabilité d'une édition papier et la vue d'ensemble des textes qu'elle offre restent irremplaçables. Les éditrices ont encore quelques années devant elles, avant d'arriver à l'édition de la période concernée, pour dépasser cette aporie. Pour l'instant, on se doit de les féliciter pour cette très belle réussite éditoriale.

\section{Balzac, l'éternelle genèse, textes réunis et présentés par Jacques Neefs, Saint-Denis, Presses universitaires de Vincennes, coll. « Manuscrits modernes », 2015, 256 p.}

\section{Compte rendu par Lionel Rerat}

L'immensité d'une œuvre ne s'établit pas à partir d'un état figé de l'écriture, du moment où le mouvement délicat de la plume cède le pas au rythme effréné des rouages métalliques d'une presse Stanhope. Sortir de la matérialité « définitive » de l'œuvre (car elle ne l'est jamais vraiment, a fortiori chez Balzac) implique que nous résistions à l'évidence de sa structuration progressive. C'est avec cette considération de l'œuvre en mouvement, façonnée et dynamisée par le regard inassouvi de son créateur, que Jacques Neefs, directeur et riche contributeur de l'ouvrage Balzac, l'éternelle genèse, pénètre l'« espace génétique polymorphe » (p. 7) que nous lègue avec fierté La Comédie humaine (1829-1855).

Appréhender l'écriture balzacienne à partir de sa genèse sera sans doute considéré comme un exercice périlleux et compte tenu de l'incomplétude des dossiers génétiques conservés - lacunaire. Fragment par fragment, bribe après bribe, d'approche en approche, les neuf contributeurs de ce collectif ${ }^{1}$ esquivent avec brio et érudition les rets de l'afféterie en formulant un commentaire aussi riche que diversifié à chaque point de leur étude. $\mathrm{Ne}$ se contentant guère d'y exposer complaisamment leurs trouvailles, c'est avec une grande finesse que ceux-ci se proposent d'étudier les nombreuses strates constitutives de l'écriture balzacienne.

Les contributeurs de l'ouvrage se plongent collectivement, mais chacun selon ses affinités respectives, dans l'univers des manuscrits de Balzac en livrant des analyses ponctuelles sur cet «édifice de mémoire » (p. 87), cette « cathédrale de papier » que constitue La Comédie humaine.

L'immensité du monument nous est donnée à voir à partir de l'analyse des «dossiers-Balzac », dossiers dont les auteurs de l'ouvrage ne manquent pas de rappeler le caractère «partial » et « incomplet». Dans la première section de l'étude, Isabelle Tournier revient sur cette « incomplétude » en nous proposant d'ailleurs une distinction intéressante entre « dossiers d'œuvre » et « dossiers génétiques », remettant ainsi en cause la nomenclature usuelle de la génétique à partir du cas Balzac. Le regretté Roger Pierrot ne manque pas de nous rappeler lui aussi au fil de sa plume que « [c]haque stratégie de classement et de conservation correspond à des attentions et à des intérêts différents, étroitement liés à l'attention portée à la création et à l'écriture » (p. 30). En lisant ce volume, nous découvrons à quel point l'entreprise balzacienne se développe à la mesure des impératifs éditoriaux (cadence, échéance, dispositif) et se déploie à partir des linéaments d'une ambition, celle de La Comédie humaine. L'archive manuscrite (allographe et autographe), les épreuves, placards, dossiers et autres missives portent en leurs seins les stigmates d'une écriture qui se nourrit de sa propre matière et qui se meut sur l'autel de son autocritique.

Roger Pierrot étudie les pratiques d'écriture de Balzac à partir de sa forme, son épitexte «privé »: quelques fragments de correspondance ayant fait l'objet d'actes de conservation spécifiques. Il nous rappelle, au besoin, que la matière épistolaire est riche en informations pour les études génétiques. Isabelle Tournier, quant à elle, nous plonge dans l'univers des manuscrits, dans ces «dossiers» d'archives tendancieux au classement discutable et sans cesse à rediscuter. Elle nous démontre que les modalités archivistiques ont un impact non négligeable sur l'interprétation de l'œuvre. Enfin, Jacques Neefs interroge La Comédie humaine dans son rapport au temps : il nous rappelle le rôle qu'a joué l'Histoire, la grande, dans le processus de création de l'œuvre dont le caractère sociologique s'est affirmé progressivement.

La deuxième section de l'ouvrage nous transporte au cœur de l'acte d'écriture balzacien, à la virtualité de son espace, sa cadence, ses habitudes, sa réflexivité. Nous découvrons ici que le moment de l'écriture coïncide avec celui de la naissance d'une œuvre pensée comme un tout anthologique. Saisie au travers de ses nombreuses mutations davantage que par ses métamorphoses -, l'écriture de Balzac se donne à voir de

1. R. Pierrot, I. Tournier, J. Neefs, C. BarelMoisan, M. Lichtlé, A. Del Lungo, E. Bordas, S. Pietri et A. Herschberg Pierrot. L'ordre nominatif suit la structure de l'ouvrage. 
prime abord comme un projet d'écriture : celui de La Comédie humaine. Michel Lichtlé, quant à lui, nous rapproche encore davantage de la pratique auctoriale en s'intéressant à l'utilisation spécifique que Balzac fait de l'épreuve, nous rendant particulièrement attentifs à ce nouveau rapport que l'auteur institue entre l'épreuve et le manuscrit, ceux-ci se confondant presque dans leurs usages. Pour terminer par le début, Andrea Del Lungo conclut cette section sur l'acte d'écriture en interrogeant les "faux départs » balzaciens. Ces «faux départs » en tant que « trace textuelle de ces débuts sans suite et répétés » (p. 148) ont le mérite de nous rendre attentifs à la contrainte que l'écriture de l'incipit représentait pour Balzac.

La troisième partie se déploie autour de trois "genèses singulières ». Éric Bordas nous introduit dans l'univers dramaturgique de Balzac, en nous proposant une analyse de la pratique scripturale relative à l'élaboration des pièces théâtrales. Susi Pietri nous invite, quant à elle, à une relecture du Chef-d' œuvre inconnu, véritable espace littéraire au sein duquel la genèse de l'écriture se superpose à la « genèse picturale » (p. 201). Souvent, les contributeurs du volume ont constaté que le canevas de La Comédie humaine transparaissait dans le corps du roman luimême, l'ambition du « tout » déteignant prospectivement sur la partie, la noblesse de la charge prévalant sur l'humeur du moment, « le travail du style se confondant avec le travail de l'œuvre » (p. 240). C'est sur cette réflexion qu'Anne Herschberg Pierrot clôt l'ouvrage ; une réflexion qu'elle conduit à partir de l'analyse des nombreux et édifiants « ajouts » qui émaillent la genèse de La Jeune Fille aux yeux d'or. En effet, si nous avons vu que Balzac accorde une importance singulière à la structure de sa Comédie humaine, nous apprenons que son unité s'établit aussi par un processus d'amplification. Les ajouts textuels dont fait l'objet ce roman de 1835 s'inscrivent pleinement dans la volonté d'unir rétrospectivement une pièce à l'ensemble de la collection.

Mon intérêt personnel pour la correspondance en tant que source historique m'a fait apprécier tout particulièrement l'article de Roger Pierrot. Il y partage succinctement mais avec enthousiasme son goût pour l'épistolaire en attirant notre regard sur les missives que l'auteur adresse à sa famille, ses éditeurs et la «bien aimée » Mme Hanska. Ces échanges épistolaires - hélas, indisponibles dans leur intégralité - nous mettent au bénéfice d'éclaircissements certains sur les projets d'écriture de Balzac ; ainsi pouvons-nous mesurer l'écart entre les ambitions premières de l'auteur et la réalité « finalisée » de l'imprimé. Dans une lettre du 26 octobre 1834 adressée à Mme Hanska, Balzac, relativement à la trilogie que constituent les Études de mours, les Études philosophiques et les Études analytiques, écrit :

Les mœurs sont le spectacle, les causes sont les coulisses et les machines. Les principes, c'est l'auteur ; mais, à mesure que l'œuvre gagne en spirale les hauteurs de la pensée, elle se resserre et se condense. S'il faut 24 volumes pour les Études de mœurs, il n'en faudra que 15 pour les Ét[udes] phil[osophiques] ; il n'en faut que 9 pour les Études analytiques. Ainsi, l'homme, la société, l'humanité seront décrits, jugés, analysés sans répétitions, et dans une œuvre qui sera comme les Mille et une Nuits de l'Occident.

Cet extrait démontrerait presque à lui seul la dimension prospective de La Comédie humaine si les phases relatives à son élaboration en étaient occultées. Fort heureusement, l'étude du « chantier » donne lieu à une réflexion sur son « architecture ».

C'est pour en parler davantage que j'ai omis de mentionner l'article de Claire Barel-Moisan qui, dans la deuxième partie du recueil, se donne pour objet d'étudier l'évolution de l'architecture de La Comédie humaine à partir des déplacements des œuvres au sein de l'ensemble.
Quelle place accorder à ces mouvements de la structure, représentent-ils « des accidents insignifiants » (p. 113) subordonnés à un « tout » qui nous aveugle ou doiventils, au contraire, être pensés comme des motifs participant à la création du « tout » lui-même ? Claire Barel-Moisan répond à cette large et ancienne problématique au travers d'une étude qui impressionne par la finesse de l'analyse autant que par la richesse de la méthode employée. Parmi les déplacements auxquels ont été sujettes de nombreuses œuvres (vingt-trois sur un total de quatre-vingt-dix), elle opère d'abord une distinction entre les déplacements « externes » (à savoir ceux qui émanent d'une décision - souvent - éditoriale et qui concernent les trois grands ensembles de La Comédie humaine) et les déplacements « internes » (procédant du choix de l'auteur). De manière très convaincante, elle classe ensuite les œuvres concernées par ces déplacements en fonction de leurs assignations à une autre édition (1830-1846) ou en fonction de leurs transpositions à d'autres « étages » de La Comédie humaine (ainsi qu'à d'autres sections). L'étude se poursuit sur les enjeux interprétatifs que lesdits déplacements impliquent et qu'ils ont engendrés alors que la critique balzacienne n'était encore qu'à ses prémices - offrant ainsi au passage une appréciable rétrospective sur le regard que portaient des critiques contemporains de Balzac tels que Félix Davin ou Alphonse du Valconseil.

Pour conclure mon parcours nécessairement sélectif, je soulignerai le caractère bienvenu de l'étude d'Éric Bordas sur la genèse théâtrale. Souvent reléguée au second plan, l'œuvre théâtrale de Balzac fait l'objet ici d'une étude rendant manifestes les nombreuses spécificités de l'écriture dramaturgique de l'auteur, et plus singulièrement les nombreuses interférences stylistiques que son écriture entretient avec le genre romanesque. Il nous incite aussi à repenser la frontière que tracent les études génétiques entre l'écriture du texte et sa mise en scène. 
Balzac, l'éternelle genèse se construit comme un triptyque de l'univers scriptural de Balzac (les trois sections de ce volume répondant aux trois parties de La Comédie humaine) que le généticien esquisse - à défaut de totaliser - à partir de la singularité d'un ajout, l'originalité d'un geste, le choix d'un lieu, d'un espace, d'une époque. C'est par la petite porte que nous entrons dans la Tétralogie balzacienne, en rendant hommage au travail de « [c] e grand peintre de fresque » qui fut « un incomparable miniaturiste ${ }^{2}$ ».

Gustave Flaubert, Scénarios de La Tentation de saint Antoine. Le temps de l'ouvre, présentation, transcription et notes par Gisèle Séginger, MontSaint-Aignan, Presses universitaires de Rouen et du Havre, 2014, 300 p.

\section{Compte rendu par Jacques Neefs}

Les manuscrits de travail de Flaubert sont, cela est maintenant bien connu, exemplaires quant à ce que l'on peut lire en eux de l'élaboration d'une " poétique » singulière, ce que Flaubert appelait la « poétique insciente » de l'œuvre, et donc du « travail de l'œuvre ». Le chantier de conception et d'écriture apparaît bien comme le lieu où se cherchent l'intensité et l'esthétique propres à chaque œuvre, comme si celle-ci projetait en avant d'elle-même son exigence - et Flaubert soulignait, à chaque fois, que l'entreprise d'une œuvre nouvelle était comme une nouvelle navigation inconnue. L'exemple de Flaubert a en cela été déterminant pour l'étude de ce que l'on peut appeler « les styles de la genèse 3 », aussi bien que pour la définition de la critique génétique et de ses apports esthétiques et épistémologiques.

Nombre de manuscrits de Flaubert sont désormais largement «publiés", selon différentes modalités d'édition, éditions imprimées et éditions numériques ${ }^{4}$. Les scénarios de La Tentation de saint Antoine deviennent à leur tour accessibles, et parfaitement lisibles, grâce à la publication qu'en fait Gisèle Séginger en ce volume. Ces scénarios et notes, ainsi présentés, constituent une passionnante source d'exploration et de réflexion sur le travail esthétique et «philosophique » que Flaubert a développé dans cette œuvre double, qui d'une certaine manière l'a accompagné « toute sa vie », comme il l'indiquait lui-même. Et cette publication permet de mesurer comment Flaubert, juste avant ce que l'on considère comme la radicale ascèse esthétique que constitue l'écriture de Madame Bovary, avait déjà, de fait, expérimenté, en concevant La Tentation de saint Antoine, des modalités d'invention aux prises avec les mythes et les croyances qui interrogent profondément les capacités de l'espace littéraire.

Cette édition se distingue nettement des éditions précédentes des scénarios de La Tentation, que ce soit celle des Euvres complètes de Flaubert du Club de l'Honnête homme (1972) ou celle qu'a donnée Young-Eun Kim dans son livre La Tentation de saint Antoine, version de 1849. Genèse et structure (Kangweon University Press, 1990). La première avait certes le mérite et peut-être pourrait-on dire l'audace éditoriale de publier ainsi scénarios et notes manuscrits accompagnant les œuvres (c'est le cas pour toutes les œuvres dans cette édition), et d'être ainsi tout à fait contemporaine de l'intérêt naissant pour les « manuscrits » préparatoires et la lecture du travail de l'écrivain. Mais, pour Saint Antoine elle répartissait sur les deux tomes correspondant à l'édition de 1874, tome IV, et aux versions de 1849 et de 1856 , tome IX, les manuscrits des scénarios et des notes préparatoires, d'une manière relativement incomplète et selon un classement que le travail de Gisèle Séginger rectifie de manière convaincante. La seconde édition ne concerne, comme indiqué par le titre, que les scénarios de la version de 1849 . Or, et c'est là que le travail de Gisèle Séginger intervient de manière tout à fait décisive et éclairante, ces « scénarios », version de 1849 et 1856 et version de 1874 , ont été réunis de manière relativement brouillée, conservés ensemble sous la cote NAF 23671 de la Bibliothèque nationale de France. Cet ensemble de manuscrits comprend également des notes et quelques brouillons des trois périodes de travail : 1846-1849, 1856, 1869-1872. Il fallait donc d'abord en débrouiller la confusion. C'est ce que Gisèle Séginger avait fait de manière précise et assurée dès son livre Naissance et métamorphoses d'un écrivain : Flaubert et les Tentations de saint Antoine ${ }^{5}$, résultat de sa thèse conduite sous la direction de Claude Duchet et dans le cadre de l'ITEM. Alors, par une réflexion sur l'esthétique en jeu dans les pages, par une analyse fine des dispositifs d'écriture, plus encore que par des confirmations codicologiques (écriture, papiers)

2. Marcel Proust, lettre à Mme de CaramanChimay, 20 juillet 1907.

3. Voir Anne Herschberg Pierrot, Le Style en mouvement. Littérature et art, chap. VI, «Styles de la genèse », Paris, Belin, 2005, p. 141-180. 4. Citons Plans et scénarios de "Madame Bovary », éd. Yvan Leclerc, Paris, CNRS Éditions/Zulma, 1995, et l'ensemble des manuscrits de Madame Bovary en ligne sur le site Flaubert de l'Université de Rouen, <http:// flaubert.univ-rouen.fr/> ; les plans et scénarios de Salammbô, transcrits par Atsuko Ogane (transcription disponible sur le site Flaubert de l'Université de Rouen) et les manuscrits de Salammbô transcrits par Geneviève JulliardMondon, dans sa thèse de doctorat, « Genèse du personnage de Salammbô d'après les manuscrits autographes de Gustave Flaubert », Université Paris VII, 2001 ; les scénarios de L'Éducation sentimentale édités par Tony Williams, Paris, Corti, 1992 ; l'ensemble des manuscrits de Trois Contes classés et transcrits par Giovanni Bonnacorso et son équipe ( $U n$ cœur simple, Paris, Les Belles Lettres, 1983 ; Hérodias, Paris, Nizet, 1991 ; La Légende de saint Julien l'Hospitalier, Paris, Didier érudition, 1998) ; les manuscrits de Bouvard et Pécuchet, classés et transcrits en ligne, dir. Yvan Leclerc, sur le site Flaubert de Rouen, et l'édition électronique des dossiers de Bouvard et Pécuchet, dir. Stéphanie DordCrouslé, <www.dossiers-flaubert.fr/>.

5. Naissance et métamorphoses d'un écrivain : Flaubert et les Tentations de saint Antoine, Paris, Honoré Champion, 1997. 